\title{
Challenging Results Make Us Think
}

\author{
Claudia M. Witt
}

Institute for Complementary and Integrative Medicine, University Hospital Zurich and University Zurich, Zurich, Switzerland

The use of complementary medicine in cancer patients is an often and controversially discussed topic in oncology. Nevertheless, for a comprehensive treatment of cancer patients, it is important that health professionals who are involved in cancer treatment are informed about the patients' interests, preferences, and current use of complementary medicine. A more open-minded approach of health professionals could positively influence the relationship between them and their patients.

In this context, the results presented by Koenig et al. [1] in this issue of Oncology Research and Treatment are quite challenging, as they contradict current evidence found in the literature. Why do I still think it is important to publish the paper? Results that don't match the available evidence and our personal professional experience usually make us think thoroughly and can motivate us to improve our research methods. The challenge the authors provide us with is that only $18 \%$ of the cancer patients who completed the questionnaire indicated that they used complementary medicine. As the authors even included prayers and relaxation techniques in their definition of complementary medicine, this percentage seems especially low. We know that even psycho-oncologists often teach relaxation techniques to cancer patients and a market research survey (GFK Marktforschung Nürnberg) in 2006 suggested that around half of the female population prays in difficult situations.

Furthermore, if the results by Koenig et al. [1] were representative for Germany, the use of complementary medicine in cancer patients would have decreased over time. A meta-analysis by Horneber et al. [2] published in 2012 in the Journal of Integrative Oncology suggested the opposite by showing an increased use of complementary medicine in cancer patients, from an estimated $25 \%$ in the 1970 s and 1980 s to more than $32 \%$ in the 1990 s and to $49 \%$ after 2000.

Because up to $15 \%$ of the data included in the meta-analysis came from surveys performed in Germany and showed that around $42 \%$ of German cancer patients used complementary medicine, I personally don't believe that the results presented by Koenig and colleagues are representative for German cancer patients. The question is whether the data reflect the complementary medicine use of the patients visiting the oncological/hematological outpatient department in Mannheim, where the survey was performed. With only $18 \%$ of respondents using complementary medicine, I have my doubts and one might speculate that non-respondents (patients who got the questionnaire but did not complete it; 58\%) used complementary medicine more often than those who completed the questionnaire (42\%). It might also be possible that the respondents were unwilling to reveal complementary medicine use, fearing that this might be regarded as undesirable.

Another explanation for possible underreporting of complementary medicine use in this survey might be that patients who completed the questionnaire may not have understood the questions correctly or the authors might not have asked all relevant questions. Complementary medicine is an especially heterogeneous field and we know from our work in the EU FP7 funded project CAMbrella (www.cambrella.eu) that survey results depend very much on the way questions are asked, on the selection of the study population and on the culture within the country itself.

For patients, but even for health professionals it is very difficult to draw a clear line and decide what is complementary medicine and what is already part of conventional cancer care or just a healthy life-style. Changes in nutrition, exercise, and relaxation are often suggested by different health professions as well as by family members or friends and many patients might not even think about those when asked to complete a questionnaire on complementary medicine.

However, those aspects could be identified when pretesting questionnaires and it is a pity that the authors didn't provide more information about the methods they used when pretesting their questionnaire and the results of their pretest. Furthermore, the authors report that the questionnaire from this study has been used in parallel in a multicenter study with more than 2,000 cancer patients and results will be available soon. Unfortunately, they don't provide information on whether they have revised their questionnaire and their recruitment strategy for the larger survey; and we can only hope that they did take the opportunity to do so.

\section{KARGER \\ Fax +497614520714

() 2016 S. Karger GmbH, Freiburg

$2296-5270 / 16 / 0395-0258 \$ 39.50 / 0$
Prof. Claudia Witt, MD, MBA

Institute for Complementary and Integrative Medicine

University Hospital Zurich and University Zurich

Sonneggstr. 6, 8091 Zurich, Switzerland

claudia.witt@uzh.ch 
All of us who regularly perform surveys should be motivated to spend more time in the future on developing and testing our survey items. Furthermore, survey authors should use the opportunity to analyze the data in a more complex way that provides information about relationships between patients' characteristics and their complementary medicine use.

To assess the use of complementary medicine in a representative manner in the future, a more sophisticated methodological approach is needed, which includes: 1) choosing clinics randomly,
2) including a large enough number of consecutive patients of each relevant cancer type and 3) focusing on those complementary medicine methods we are mainly interested in and patients can identify, because sometimes less can be more.

\section{Disclosure Statement}

The author declares to have no conflicts of interest.

\section{References}

König J, Geschwill K, Lang A, Tauchert FK, Hofheinz $\mathrm{RD}$, Kripp M: Use of complementary and alternative medicine in cancer patients. Oncol Res Treat 2016; DOI: $10.1159 / 000446008$.
Horneber M, Bueschel G, Dennert G, Less D, Ritter E, Zwahlen M: How many cancer patients use complementary and alternative medicine: a systematic review and metaanalysis. Integr Cancer Ther 2012;11:187-203. 